\title{
THE HADAMARD CONJECTURE AND INTEGER LATTICES
}

\author{
J. McCALL and C. H. C. LITTLE \\ (Received 20 January 1985; revised 25 August 1986) \\ Communicated by W. D. Wallis
}

\begin{abstract}
Let $L$ be an integer lattice, and $S$ a set of lattice points in $L$. We say that $S$ is optimal if it minimises the number of rectangular sublattices of $L$ (including degenerate ones) which contain an even number of points in $S$. We show that the resolution of the Hadamard conjecture is equivalent to the determination of $|S|$ for an optimal set $S$ in a $(4 s-1) \times(4 s-1)$ integer lattice $L$. We then specialise to the case of $1 \times n$ integer lattices, characterising and enumerating their optimal sets.
\end{abstract}

1980 Mathematics subject classification (Amer. Math. Soc.): 05 B 20.

\section{Introduction}

The well-known Hadamard conjecture asserts the existence of a $4 s \times 4 s$ Hadamard matrix for each positive integer $s$. In [2] its resolution is shown to be equivalent to the discovery of a basis for the cycle space of the complete bipartite graph $K_{4 s, 4 s}$ which maximises the number of 4-circuits which are the sum (modulo 2) of an odd number of basis circuits. Of course, the search for such a basis can be conducted on other graphs as well. For $K_{m, n}$ with $m \geqslant 2$ and $n \geqslant 2$, we use a basis studied by Schmeichel in [4] to transform the search into a problem about an $(m-1) \times(n-1)$ integer lattice. A thorough study of this problem is then made for the case $m=2$.

(c) 1987 Australian Mathematical Society 0263-6115/87 \$A2.00+0.00 


\section{The relationship between the Hadamard conjecture and integer lattices}

Let $m \geqslant 2$ and $n \geqslant 2$. Let $K_{m, n}$ be the complete bipartite graph with vertex set $\left\{x_{1}, x_{2}, \ldots, x_{m}, y_{1}, y_{2}, \ldots, y_{n}\right\}$, where $x_{i}$ is adjacent to $y_{j}$ for each $i$ and $j$. Any 4-circuit in $K_{m, n}$ will be denoted by listing its vertices as a string. Let $S$ be the set of $(m-1)(n-1)$ 4-circuits of the form $x_{i} y_{j} x_{i+1} y_{j+1}$ where $1 \leqslant i \leqslant m-1$ and $1 \leqslant j \leqslant n-1$. It is shown in [4] that $S$ is a basis of the cycle space of $K_{m, n}$. Moreover, if $x_{r} y_{s} x_{t} y_{u}$ is a 4-circuit, where $r<t$ and $s<u$, then

$$
x_{r} y_{s} x_{t} y_{u}=\sum_{j=s}^{u-1} \sum_{i=r}^{t-1} x_{i} y_{j} x_{i+1} y_{j+1}
$$

where the sum of circuits is determined by the symmetric difference of their edge sets.

The idea propounded in this paper is to represent the 4-circuit $x_{i} x_{j} x_{i+1} y_{j+1}$ by the point in the plane with coordinates $(i, j)$. Given positive integers $a, b, c, d$ such that $a \leqslant c$ and $b \leqslant d$, let us define $R(a, b, c, d)$ as the set of all points with coordinates of the form $(i, j)$ where $a \leqslant i \leqslant c, b \leqslant j \leqslant d$ and $i$ and $j$ are integers. We call $R(a, b, c, d)$ a rectangle, even though it may consist of a single point, in which case we deem it to be doubly degenerate. The points $(a, b),(a, d)$, $(c, b),(c, d)$ are the corners of the rectangle. By (1) we see that the 4-circuit $x_{r} y_{s} x_{t} y_{u}$, where $r<t$ and $s<u$, is the sum of those represented by the elements of $R(r, s, t-1, u-1)$. Thus we may conceive of $x_{r} y_{s} x_{t} y_{u}$ as being represented by $R(r, s, t-1, u-1)$. The doubly degenerate rectangles therefore represent the circuits in $S$. We shall identify the circuits in $S$ with the corresponding points.

Let us now introduce an arbitrary basis $B$ of the cycle space of $K_{m, n}$. We must inquire as to whether $x_{r} y_{s} x_{t} y_{u}$ is the sum of an even or an odd number of circuits in $B$. Let $S_{0}$ and $S_{1}$ be complementary subsets of $S$. Suppose that the points in $S_{0}$ and $S_{1}$ represent circuits in $S$ that are the sum of an even or an odd number, respectively, of circuits in $B$. Then the circuit represented by any rectangle $R$ is the sum of an odd number of circuits of $B$ if and only if $\left|R \cap S_{1}\right|$ is odd. One problem which emerges is the following: given $S_{1}$, can a suitable $B$ be found? The following theorem reveals the answer. Let us first say that $B$ is $S_{1}$-primitive if every circuit in $S_{1}$ is the sum of an odd number of circuits in $B$ and every circuit in $S_{0}$ is the sum of an even number of circuits of $B$.

TheOREM 1. Let $S_{1} \subseteq S$. Then there is an $S_{1}$-primitive basis $B$ of the cycle space of $K_{m, n}$ if and only if $S_{1} \neq \varnothing$.

Proof. Suppose that $S_{1}=\varnothing$, and that there exists an $S_{1}$-primitive basis $B$ of the cycle space $\mathscr{C}$ of $K_{m, n}$. Then every circuit in $S$ is the sum of an even number of circuits in $B$. As $S$ is a basis of $\mathscr{C}$, it follows that every circuit of $K_{m, n}$ is the 
sum of an even number of circuits in $B$. As the circuits in $B$ provide counterexamples to this assertion, we infer that $B$ does not exist.

Suppose $S_{1} \neq \varnothing$. Let $L$ be the integer lattice of points of the form $(i, j)$ where $i, j$ are integers such that $1 \leqslant i \leqslant m-1$ and $1 \leqslant j \leqslant n-1$.

Observe first that $L$ may be partitioned into rectangles containing exactly one point of $S_{1}$. For example, choose any point $p \in S_{1}$, and let $p=(i, j)$. If there is no positive integer $h<i$ such that $(h, j) \in S_{1}$, then let $a=1$. Otherwise let $h$ be the largest integer less than $i$ for which $(h, j) \in S_{1}$, and let $a=h+1$. If there is no integer $h^{\prime}>i$ such that $\left(h^{\prime}, j\right) \in S_{1}$, then let $c=m-1$; otherwise let $c=i$. If there is no positive integer $k<j$ such that $(l, k) \in S_{1}$ for some $l$, then let $b=1$. Otherwise let $k$ be the largest integer less than $j$ for which $(l, k) \in S_{1}$ for some $l$, and let $b=k+1$. If there is no integer $k^{\prime}>j$ such that $\left(l, k^{\prime}\right) \in S_{1}$ for some $l$, then let $d=n-1$; otherwise let $d=j$. Let $r(p)=R(a, b, c, d)$. It is now clear that the set $\mathscr{P}$ of all rectangles of the form $r(p)$, where $p \in S_{1}$, constitutes the desired partition of $L$.

We must construct an $S_{1}$-primitive basis $B$ of $\mathscr{C}$. The circuits in $B$ will be those that are represented by a rectangle which is contained in a rectangle in $\mathscr{P}$ and has a point in $S_{1}$ as one of its corners.

In order to show that $B$ is a basis, we first enumerate its elements. Choose any point $(a, b)$ in $L$. This point belongs to a unique rectangle $P \in \mathscr{P}$. This rectangle in turn contains a unique point $(i, j) \in S_{1}$. Let $m_{1}=\min (a, i), m_{2}=\min (b, j)$, $M_{1}=\max (a, i), M_{2}=\max (b, j)$. Then $R\left(m_{1}, m_{2}, M_{1}, M_{2}\right)$ is a rectangle $f(a, b)$ representing a circuit in $B$. This function $f$ is surjective, for if $R$ is any rectangle representing a circuit in $B$, then just one of its corners is a point in $S_{1}$, and $R$ is the image of the diagonally opposite corner. $R$ is not the image of any other point, and so $f$ is injective. Hence $|B|$ is equal to the number of points in $L$, which is $(m-1)(n-1)$, the dimension of $\mathscr{C}$.

Certainly each point in $S_{1}$ represents a circuit in $B$. It therefore suffices to show that each point in $S_{0}$ represents a circuit which can be written as the sum of an even number of circuits in $B$.

We therefore suppose that the point $(a, b)$ chosen above belongs to $S_{0}$. Without loss of generality, we may assume that either $a<i$ and $b=j$, or $a<i$ and $b<j$. In the former case, it follows from (1) that the circuit $C$ represented by $(a, b)$ is the sum $x_{a} y_{j} x_{i+1} y_{j+1}+x_{a+1} y_{j} x_{i+1} y_{j+1}$. Similarly in the latter case, we have

$$
C=x_{a} y_{b} x_{i+1} y_{j+1}+x_{a+1} y_{b} x_{i+1} y_{j+1}+x_{a} y_{b+1} x_{i+1} y_{j+1}+x_{a+1} y_{b+1} x_{i+1} y_{j+1} \text {. }
$$

Hence $B$ is the required $S_{1}$-primitive basis.

Let $L$ be the integer lattice introduced in the above proof. We have $L \neq \varnothing$ because $m \geqslant 2$ and $n \geqslant 2$. We now define a subset $S_{1}$ of $S$ to be an optimal set for $L$ if the number of rectangles $R$ in $L$ for which

$$
\left|R \cap S_{1}\right| \equiv 1 \bmod 2
$$

is maximised. We note the following result. 
THEOREM 2. If $S_{1}$ is optimal, then $S_{1} \neq \varnothing$.

Proof. If $S_{1}=\varnothing$, then (2) holds for no rectangle $R$. If $S_{1} \neq \varnothing$, then (2) holds for any doubly degenerate rectangle consisting of a point of $S_{1}$. Hence $S_{1}$, if empty, cannot be optimal.

From Theorems 1 and 2 we see that for any optimal set $S_{1}$ there is an $S_{1}$-primitive basis. It therefore follows from the remarks in the introduction that the resolution of the Hadamard conjecture is equivalent to the calculation of the number of rectangles satisfying (2) where $S_{1}$ is an optimal set for a $(4 s-1) \times$ $(4 s-1)$ integer lattice. This observation motivates the study of optimal sets. In the next section we characterise the optimal sets for a $1 \times n$ integer lattice.

\section{Characterisation of optimal sets for a $1 \times n$ integer lattice}

The problem of finding an optimal set is equivalent to that of finding a set $S_{1}$ such that the number of rectangles $R$ in $L$ for which

$$
\left|R \cap S_{1}\right| \equiv 0 \bmod 2
$$

is minimised. For a $1 \times n$ integer lattice $L$, let $\gamma(n)$ be the minimum possible number of rectangles $R$ in $L$ for which (3) holds. The first task is to compute $\gamma(n)$.

THEOREM 3. Let $L$ be a $1 \times n$ integer lattice. If $n$ is even, then $\gamma(n)=n^{2} / 4$. If $n$ is odd, then $\gamma(n)=\left(n^{2}-1\right) / 4$.

Proof. For each $S_{1} \subseteq L$, let $T\left(S_{1}\right)$ be the set of rectangles $R$ for which (3) holds.

Let $L=\left\{p_{1}, p_{2}, \ldots, p_{n}\right\}$ where $p_{i}=(i, 1)$ for all $i$. These points together with $p_{n+1}=(n+1,1)$ form the vertex set of a copy of the complete graph $K_{n+1}$. For simplicity we denote the rectangle $R(i, 1, j, 1)$ by $R(i, j)$ for each $i$ and $j$. This rectangle shall correspond in $K_{n+1}$ to the edge joining vertices $p_{i}$ and $p_{j+1}$. If $i \leqslant j<k$, then at least one of $R(i, j), R(j+1, k), R(i, k)$ must be in $T\left(S_{1}\right)$. The corresponding edges of $K_{n+1}$ form a triangle. Hence $\left|T\left(S_{1}\right)\right|$ is at least as large as the minimum number of edges whose deletion from $K_{n+1}$ yields a graph with no triangles. By a theorem of Turán (see [5] or [1]), the largest subgraph of $K_{n+1}$ having no triangles is $K_{n / 2,(n+2) / 2}$ if $n$ is even and $K_{(n+1) / 2,(n+1) / 2}$ if $n$ is odd. The numbers of edges in these graphs are $n(n+2) / 4$ and $(n+1)^{2} / 4$ respectively. Hence the numbers of edges in their complements are $n^{2} / 4$ and $\left(n^{2}-1\right) / 4$ respectively. We infer that $\gamma(n) \geqslant n^{2} / 4$ if $n$ is even, and $\gamma(n) \geqslant$ $\left(n^{2}-1\right) / 4$ if $n$ is odd. 
In order to establish the required equations, take $S_{1}=L$. The number of rectangles for which (3) holds is now easily seen to be $\sum_{k=1}^{n / 2}(n-2 k+1)=n^{2} / 4$ if $n$ is even and $\sum_{k=1}^{n-1) / 2}(n-2 k+1)=\left(n^{2}-1\right) / 4$ if $n$ is odd. The theorem follows.

For simplicity we identify each lattice point with its abscissa. For the rectangle $R(i, j)$ as defined in the above proof, we call $i$ the origin and $j$ the terminus. $R$ will be called odd or even according to whether it satisfies (2) or (3). For each integer $p$ such that $1 \leqslant p \leqslant n$ it is convenient to know how many even rectangles have $p$ as terminus. The following lemma supplies easy recursion for the answer.

LeMMA 4. Let $L$ be $a 1 \times n$ integer lattice. For each integer $p \in\{1, \ldots, n\}$, let $e(p, L)$ denote the number of even rectangles of $L$ with terminus $p$. Then

$$
e(1, L)= \begin{cases}1 & \text { if } 1 \in S_{0} \\ 0 & \text { if } 1 \in S_{1}\end{cases}
$$

(b) for each $p>L$,

$$
e(p, L)= \begin{cases}e(p-1, L)+1 & \text { if } p \in S_{0} \\ p-e(p-1, L)-1 & \text { if } p \in S_{1} .\end{cases}
$$

Proof. (a) Obvious.

(b) If $p \in S_{0}$, the even rectangles with terminus $p$ are $\{p\}$ and those of the form $R \cup\{p\}$ where $R$ is an even rectangle terminating at $p-1$. Suppose therefore that $p \in S_{1}$. Then the even rectangles with terminus $p$ are those of the form $R^{\prime} \cup\{p\}$ where $R^{\prime}$ is an odd rectangle terminating at $p-1$. There are $p-1-e(p-1, L)$ such rectangles.

We call $e(p, L)$ the contribution of $p$ to $\Gamma(L)$, where $\Gamma(L)$ is the number of rectangles $R$ in $L$ for which (3) holds. Thus $\Gamma(L) \geqslant \gamma(n)$, with equality if and only if $S_{1}$ is optimal. In this case, we sometimes refer to $L$ itself as optimal.

LeMMA 5. Let $L$ and $M$ be $1 \times n$ and $1 \times(n-2)$ integer lattices respectively, where $n>2$. If $\Gamma(L)-\Gamma(M)=n-1$, then $M$ is optimal if and only if $L$ is optimal.

Proof. Suppose $L$ is optimal. If $n$ is even, then

$$
\Gamma(M)=\Gamma(L)-n+1=\frac{n^{2}}{4}-n+1=\frac{(n-2)^{2}}{4},
$$

whereas if $n$ is odd then

$$
\Gamma(M)=\frac{n^{2}-1}{4}-n+1=\frac{(n-2)^{2}-1}{4} .
$$

In both cases $M$ is optimal. Similarly if $M$ is optimal then so is $L$. 
We are now prepared for our characterisation of $1 \times n$ optimal integer lattices. It is convenient here to introduce the empty integer lattice $\Lambda$, which we consider to be optimal. We also specify $S_{1}$ by writing the characteristic function of $L \cap S_{1}$, which we represent by a string of 0 's and 1's. Thus the $i$ th element of this string is 0 if $i \in S_{0}$ and 1 otherwise.

THEOREM 6. Let L be $a 1 \times n$ integer lattice. Then $L$ is optimal if and only if one of the following conditions obtains

(a) $L \in\{\Lambda, 1,10\}$;

(b) $L=M 11 N$ where $M N$ is an optimal $1 \times(n-2)$ integer lattice;

(c) $L=M 010 N$ where $M 1 N$ is an optimal $1 \times(n-2)$ integer lattice;

(d) $L=M 01$ where $M$ is an optimal $1 \times(n-2)$ integer lattice.

Moreover if $L$ is optimal then $N$ in (b) and (c) may be chosen to contain only 0 's.

Proof. We show first that if one of the above conditions holds, then $L$ is optimal.

(a) Obvious.

(b) We need to compute $\Gamma(L)-\Gamma(M N)$. Let $x$ be the contribution to $\Gamma(M N)$ of the last element of $M$. (We take $x=0$ if $M=\Lambda$.) Let $N$ be a $1 \times m$ integer lattice. The respective contributions to $\Gamma(L)$ of the two new 1's are $n-m-2-x$ and $n-m-1-(n-m-2-x)=x+1$. The contribution $e(n-m+1, L)$ to $\Gamma(L)$ of the first element of $N$, if it exists, is therefore $x+2=(x+1)+1=$ $e(n-m-1, M N)+1$ if $n-m+1 \in S_{0}$ in $L$, and $n-m-(x+1)=$ $(n-m-x-2)+1=e(n-m-1, M N)+1$ if $n-m+1 \in S_{1}$ in $L$. Thus the first point of $N$ contributes 1 more to $\Gamma(L)$ than to $\Gamma(M N)$, and by induction so does each subsequent point. Hence $\Gamma(L)-\Gamma(M N)=(n-m-2-x)+$ $(x+1)+m=n-1$, and so $L$ is optimal by Lemma 5 .

(c) Let $x$ be the contribution to $\Gamma(M 1 N)$ of the last element of $M$. Let $N$ be a $1 \times m$ integer lattice. We now have $e(n-m-2, M 010 N)=x+1$, $e(n-m-1, M 010 N)=(n-m-2)-(x+1)=n-m-x-3$ and $e(n-m, M 010 N)=n-m-x-2$, whereas $e(n-m-2, M 1 N)=n-m-$ $x$ - 3. Hence as before each element of $N$ contributes 1 more to $\Gamma(L)$ than to $\Gamma(M 1 N)$. Thus $\Gamma(L)-\Gamma(M 1 N)=n-1$.

(d) Let $e(n-2, L)=x$. Then $e(n-1, L)=x+1$ and $e(n, L)=n-x-2$, so that $\Gamma(L)-\Gamma(M)=x+1+n-x-2=n-1$.

Therefore in cases (b)-(d), $L$ is optimal by Lemma 5 .

Conversely, suppose $L$ is optimal. We distinguish two cases.

Case I. Suppose some point of $L-\{1\}$ belongs to $S_{1}$.

A. Suppose $L$ is of the form $M 11$. By the computation above we have $\Gamma(L)-\Gamma(M)=n-1$. Therefore $M$ is optimal by Lemma 5, and so condition (b) holds. 
B. Similarly if $L$ is of the form $M 01$, then $M$ is optimal, and so condition (d) holds.

C. In the remaining subcase, the last element of $L$ is 0 . By assumption the last 1 has a predecessor, which may be 0 or 1 . In the former case, $L$ is of the form $M 010 N$ where $N$ contains only 0's. By Lemma 5 we deduce that $M 1 N$ is optimal, and so condition (c) holds. In the latter case $L$ is of the form $M 11 N$ where $N$ contains only 0 's. Here $M N$ is optimal and condition (b) holds.

Case II. Suppopse that all points of $L$, except possibly the first, belong to $S_{0}$. If $S_{0}=L$, then $\Gamma(L)=\sum_{k=1}^{n} k=n(n+1) / 2$. Since $L$ is optimal, it follows that $n(n+1) / 2 \leqslant n^{2} / 4$, so that $n(n+2) \leqslant 0$. Hence $n=0$, and so $L=\Lambda$.

Suppose therefore that $1 \in S_{1}$. (Hence $L \neq \Lambda$.) In this case $\Gamma(L)=\sum_{k=1}^{n-1} k=$ $n(n-1) / 2$, and by the argument above we obtain $n(n-2) \leqslant 0$. Thus $n \in\{1,2\}$ and so $L \in\{1,10\}$.

Theorem 6 gives a method for generating every optimal integer lattice from the set $\{\Lambda, 1,10\}$. It transpires that any optimal lattice may be derived uniquely from one of these three integer lattices by means of the operations implicit in (b)-(d), with $N$ in (b) and (c) constained to consist entirely of 0 's.

THEOREM 7. Let $L$ be an optimal $1 \times n$ integer lattice. Then $L$ is derived in a unique way from an integer lattice in $\{\Lambda, 1,10\}$ by means of the operations in (b)-(d) of Theorem 6 applied with $N$ as a string of 0's in (b) and (c).

Proof. By Theorem 6 , there exists a sequence $\mathscr{L}$ of integer lattices $L_{0}, L_{1}, \ldots, L_{q}$ such that $L_{0} \in\{\Lambda, 1,10\}, L_{q}=L$ and, for each $i>0, L_{i}$ is obtained from $L_{i-1}$ by one of the operations (b)-(d) of Theorem 6. With $N$ as hypothesised, the nature of this operation can be inferred from the form of $L_{i}$, and so $\mathscr{L}$ is uniquely determined.

Operation (d) of Theorem 6 needs to be performed at most once if $N$ is allowed to be arbitrary. This can be seen most easily if we consider the application of operations (b)-(d) in reverse in order to reduce an optimal integer lattice $L$ to an integer lattice in the set $\{\Lambda, 1,10\}$. Operations (b) and (c) may be applied repeatedly in order to obtain a string in which every element, except possibly the first or last, is 0 . Then operation (d) can be applied in reverse at most once. In fact we may dispense with it by rephrasing the theorem as follows.

THEOREM 8. Let $L$ be a $1 \times n$ integer lattice. Then $L$ is optimal if and only if one of the following conditions obtains

(a) $L \in\{\Lambda, 1,10,01,101,1001\}$;

(b) $L=M 11 N$ where $M N$ is an optimal $1 \times(n-2)$ integer lattice;

(c) $L=M 010 N$ where $M 1 N$ is an optimal $1 \times(n-2)$ integer lattice. 
This theorem follows from the above remarks together with the observation that the last three elements of the set displayed in (a) are obtained from the first three respectively by applying operation (d).

Note that a $1 \times n$ integer lattice can be tested for optimality very quickly by applying operations (b) and (c) in reverse as often as possible and then checking to see whether the resulting string is one of those specified in (a).

We conclude this section by noting that the optimal $1 \times n$ integer lattices are the elements of the context-free language, with alphabet $\{0,1\}$, generated from a symbol $S$ by the following productions:

$$
\begin{aligned}
S & \rightarrow P \\
S & \rightarrow P 1 \\
S & \rightarrow P 0 P 1 \\
S & \rightarrow P 1 P 0 P \\
S & \rightarrow P 1 P 0 P 1 \\
S & \rightarrow P 1 P 0 P 0 P 1 \\
P & \rightarrow P 1 P 1 \\
P 1 & \rightarrow P 0 P 1 P 0 P \\
P & \rightarrow \Lambda .
\end{aligned}
$$

\section{Enumeration of optimal integer lattices}

Theorem 6 can be used to enumerate optimal $1 \times n$ integer lattices for any $n$. For each $n$ and $j \leqslant n$ define $l_{n j}$ to be the number of optimal $1 \times n$ integer lattices which end with a run of exactly $j 0$ 's. The number of optimal $1 \times n$ integer lattices is $\sum_{k=0}^{n} l_{n k}$. Operations (b) (with $N=\Lambda$ ) and (d) of Theorem 6 can be used to generate distinct optimal integer lattices ending with 1 . Hence $l_{n 0}=2 \sum_{k=0}^{n-2} l_{n-2, k}$ for each $n>1$. Now let $j>0$. An optimal integer lattice ending with a run of exactly $j 0$ 's can be produced by operation (c) from one ending in a run of exactly $j-10$ 's, or by operation (b) from one ending in a run of at least $j 0$ 's. Therefore $l_{n j}=\sum_{k=j-1}^{n-2} l_{n-2, k}$. These results, together with the equations $l_{00}=l_{10}=1$ and $l_{11}=0$, enable us to obtain $l_{n j}$ recursively for any $n$. In fact, we obtain the following theorem.

THEOREM 9. If $n=2 r$ or $n=2 r+1$, there are $\left(\begin{array}{c}2 r+1 \\ r\end{array}\right)$ optimal $1 \times n$ integer lattices.

Proof. First we note the identity

$$
\sum_{k=0}^{j}\left(\begin{array}{c}
r-k \\
j-k
\end{array}\right)=\left(\begin{array}{c}
r+1 \\
j
\end{array}\right)
$$


where $r \geqslant j$. It certainly holds for all $r \geqslant j$ if $j=0$. If it holds for a fixed $j \geqslant 0$ and for all $r \geqslant j$, then for all $r>j$ we have

$$
\begin{aligned}
\sum_{k=0}^{j+1}\left(\begin{array}{c}
r-k \\
j+1-k
\end{array}\right) & =\left(\begin{array}{c}
r \\
j+1
\end{array}\right)+\sum_{k=1}^{j+1}\left(\begin{array}{c}
r-k \\
j+1-k
\end{array}\right) \\
& =\left(\begin{array}{c}
r \\
j+1
\end{array}\right)+\sum_{k=0}^{j}\left(\begin{array}{c}
r-1-k \\
j-k
\end{array}\right) \\
& =\left(\begin{array}{c}
r \\
j+1
\end{array}\right)+\left(\begin{array}{c}
r \\
j
\end{array}\right) \\
& =\left(\begin{array}{c}
r+1 \\
j+1
\end{array}\right),
\end{aligned}
$$

and so (4) holds in general whenever $r \geqslant j$.

Note also that if $j>r$ where $n=2 r+1$, then $l_{n j}=0$ by Theorem 8 . We show next that $l_{n j}=\left(\begin{array}{c}2 r-j \\ r-j\end{array}\right)$ for all $j \leqslant r$. This result certainly holds if $r=0$, since $l_{10}=1$. Assume therefore that it holds for $1 \times(n-2)$ optimal integer lattices, and that $r>0$. If $1 \leqslant j \leqslant r$, then

$$
\begin{aligned}
l_{n j} & =\sum_{k=j-1}^{n-2} l_{n-2, k} \\
& =\sum_{k=j-1}^{r-1}\left(\begin{array}{c}
2 r-k-2 \\
r-k-1
\end{array}\right) \\
& =\sum_{k=0}^{r-j}\left(\begin{array}{c}
2 r-k-j-1 \\
r-k-j
\end{array}\right) \\
& =\left(\begin{array}{c}
2 r-j \\
r-j
\end{array}\right)
\end{aligned}
$$

by (4), since $r \geqslant 1$ so that $2 r-j-1 \geqslant r-j$. Finally

$$
\begin{aligned}
l_{n 0} & =2 \sum_{k=0}^{n-2} l_{n-2, k} \\
& =2 \sum_{k=0}^{r-1}\left(\begin{array}{c}
2 r-k-2 \\
r-k-1
\end{array}\right) \\
& =2\left(\begin{array}{c}
2 r-1 \\
r-1
\end{array}\right) \quad(\text { by }(4)) \\
& =\left(\begin{array}{c}
2 r-1 \\
r-1
\end{array}\right)+\left(\begin{array}{c}
2 r-1 \\
r
\end{array}\right) \\
& =\left(\begin{array}{c}
2 r \\
r
\end{array}\right) .
\end{aligned}
$$


The number of optimal $1 \times n$ integer lattices, where $n=2 r+1$, is therefore

$$
\sum_{k=0}^{n} l_{n k}=\sum_{k=0}^{r}\left(\begin{array}{c}
2 r-k \\
r-k
\end{array}\right)=\left(\begin{array}{c}
2 r+1 \\
r
\end{array}\right)
$$

by (4).

If $n=2 r$, note first that $l_{00}=l_{10}=1$. Suppose that for all $j \leqslant n$ we have $l_{n j}=l_{n+1, j}$. Then $l_{n+2,0}=2 \sum_{k=0}^{n} l_{n k}=2 \sum_{k=0}^{n+1} l_{n+1, k}=l_{n+3,0}$ and similarly $l_{n+2, j}$ $=l_{n+3, j}$ for all $j>0$.

Therefore $l_{n j}=l_{n+1, j}$ holds by induction for all even $n$ and for all $j \leqslant n$. Hence $\sum_{k=0}^{n} l_{n k}=\sum_{k=0}^{n+1} l_{n+1, k}$, and it follows that the number of $1 \times 2 r$ optimal integer lattices is also $\left(\begin{array}{c}2 r+1 \\ r\end{array}\right)$.

Theorem 9 suggests that there may be a $1: 1$ correspondence between optimal $1 \times(2 r+1)$ integer lattices and selections of $r$ objects from a set of cardinality $2 r+1$. Like the construction of the optimal integer lattice itself, such a selection may in fact be achieved inductively as follows.

When operation (b) of Theorem 6 is performed with $N=\Lambda$, take the complement of the existing selection, and choose neither of the two new 1's. When $N \neq \Lambda$, take the existing selection together with the first of the new 1's. When operation (c) is performed, take the existing selection together with the first of the new 0's. When operation (d) is performed, take the existing selection and the new 1. A detailed proof that the resulting correspondence is $1: 1$ appears in [3].

\section{Conclusion}

Knowing from [2] that the resolution of the Hadamard conjecture is equivalent to the discovery of a basis for the cycle space of $K_{4 s, 4 s}$ which maximises the number of 4-circuits which are the sum (modulo 2) of an odd number of basis circuits, we used a $(4 s-1) \times(4 s-1)$ integer lattice as a tool to study a condition for a 4-circuit to be such a sum. Specifically, if $S$ is as defined in Section 2, and $S_{1}$ is the subset of $S$ consisting of all the circuits in $S$ which are the sum of an odd number of circuits in an arbitrary basis $B$ of the cycle space of $K_{4 s, 4 s}$, then a circuit represented by a rectangle $R$ in the integer lattice is the sum of an odd number of circuits of $B$ if and only if $\left|R \cap S_{1}\right|$ is odd. Thus we defined a subset $T$ of $S$ to be optimal for the integer lattice if the number of rectangles $R$ satisfying $|R \cap T| \equiv 1(\bmod 2)$ is maximised or, equivalently, if the number of rectangles $R$ satisfying $|R \cap T| \equiv 0(\bmod 2)$ is minimised. By Theorem 1 and Theorem 2 we saw that any optimal $T$ is of the form $S_{1}$ for some basis $B$ of the cycle space of $K_{4 s, 4 s}$. Therefore the Hadamard conjecture would be resolved by the construction of an optimal set for a $(4 s-1) \times(4 s-1)$ integer lattice. This 
observation provided the motivation for a study of the optimal sets for the simper case of a $1 \times n$ integer lattice. These optimal sets were characterised and enumerated, and constructions for them were given. It is the hope of the authors that these results will be found to provide useful tools for the study of optimal sets in larger integer lattices.

\section{References}

[1] F. Harary, Graph theory (Addison-Wesley, London, 1969), p. 17.

[2] C. H. C. Little and M. D. Hendy, 'The Hadamard conjecture and bases for the cycle space of $K_{4 s, 4 s}$ ', Ars. Combin. 17A (1984), 231-240.

[3] J. McCall, Some properties of bases for the cycle spaces of complete bipartite graphs (master's thesis, Massey University, 1984).

[4] E. F. Schmeichel, 'The basis number of a graph', J. Combin. Theory 30 (1981), 123-129.

[5] P. Turán, 'Eine Extremalaufgabe aus der Graphentheorie', Mat. Fiz. Lapok. 49 (1941), 436-452.

Department of Mathematics and Statistics

Massey University

Palmerston North

New Zealand 